\title{
Clinical Evidence and Risk Factors for Reproductive Disorders Caused by Bacterial Infections in Meat Goats in Northeastern Thailand
}

\author{
Sarinya Rerkyusuke $\mathbb{D}^{1,2}$ Sawarin Lerk-u-suke $\mathbb{D}^{3,4}$ and Anucha Sirimalaisuwan ${ }^{5}$ \\ ${ }^{1}$ Division of Livestock Medicine, Faculty of Veterinary Medicine, Khon Kaen University, Khon Kaen 40002, Thailand \\ ${ }^{2}$ Research Group of Animal Health Technology, Faculty of Veterinary Medicine, Khon Kaen University, \\ Khon Kaen 40002, Thailand \\ ${ }^{3}$ Department of Geographic Information Science, School of Information and Communication Technology, University of Phayao, \\ Phayao 56000, Thailand \\ ${ }^{4}$ Research Unit of Spatial Innovation Development, School of Information and Communication Technology, University of Phayao, \\ Phayao 56000, Thailand \\ ${ }^{5}$ Department of Veterinary Bioscience and Veterinary Public Health, Faculty of Veterinary Medicine, Chiang Mai University, \\ Chiang Mai 50100, Thailand
}

Correspondence should be addressed to Sarinya Rerkyusuke; sarinyare@kku.ac.th

Received 5 December 2021; Accepted 19 January 2022; Published 8 February 2022

Academic Editor: Arnab Sen

Copyright (C) 2022 Sarinya Rerkyusuke et al. This is an open access article distributed under the Creative Commons Attribution License, which permits unrestricted use, distribution, and reproduction in any medium, provided the original work is properly cited.

\begin{abstract}
The objective of this study was to identify risk factors related to reproductive disorders caused by bacterial infections in goats in northeastern Thailand. Two hundred twenty farms were investigated, and 49 herds were found to have clinical reproductive disorders. Moreover, 96\% (47/49) of herds showing clinical reproductive failure preferred to circulate bucks between herds. A total of 118 sera, including 85 clinical reproductive disorder cases such as abortion $(n=70)$, abortion with arthritis $(n=1)$, orchitis $(n=3)$, repeat breeder $(n=6)$, sterile $(n=1)$, and weak kids $(n=4)$, and 33 bucks' circulations were serologically tested for bacterial infections caused by Coxiella burnetii, Chlamydophila abortus, and Brucella spp. Results showed 69\% (81/118 cases) were seropositive for Q fever $(n=55 ; 46.61 \%)$, brucellosis $(n=8 ; 6.78)$, and chlamydiosis $(n=18 ; 15.25 \%)$, respectively; $82 \%$ of herds ( 40 / 49 herds) were infected with at least one of those diseases. Moreover, $40 \%$ of infected herds (16/40) had coinfection among the three of those diseases. Approximately 60\% (20/33) of buck circulation showed seropositivity to at least one of the diseases, and $85 \%$ of infected bucks were seropositive for $\mathrm{Q}$ fever (17/20). Buck circulation between herds is a risk factor for diseases on farms ( $p=0.001)$; odds ratio $(\mathrm{OR}=109.29 ; 95 \%$ confidence interval $(\mathrm{CI})=6.61-1,807.38)$. Moreover, the annual brucellosis test is a protective factor against reproductive failure cases on farms $(p=0.022$; OR $=0.45 ; 95 \% \mathrm{CI}=0.23-0.89)$. Reproductive disorder cases can be caused by sexual transmission, so buck circulation can yield Q fever, brucellosis, and chlamydiosis in communities. This investigation is the first report of chlamydiosis infection in our area. Concerning Q fever, chlamydiosis, and brucellosis are zoonotic diseases that impact animal health and production losses. Control and prevention measures related to risk factors together with active surveillance programs should be incorporated into client education.
\end{abstract}

\section{Introduction}

Bacterial infection-induced reproductive disorders, such as brucellosis, Q fever, enzootic abortion (chlamydiosis), caseous lymphadenitis (CLA), listeriosis, campylobacteriosis, leptospirosis, salmonellosis can be found in Caprinae [1].
Q fever is caused by Gram-negative bacteria called Coxiella burnetii. As for clinical signs, does and nannies show abortions in the first trisemester, stillbirths, a retained placenta, endometritis, infertility, weak kids, and repeat breeding [2]. Chlamydiosis is caused by Gram-negative bacteria called Chlamydophila abortus. Infected doe goats 
TABLE 1: Description of clinical reproductive disorders and bucks' circulation related to seropositivity for Q fever, chlamydiosis, and brucellosis in 49 smallholder goat herds in northeastern Thailand.

\begin{tabular}{lcccc}
\hline Reproductive disorder & Case $(n)$ & Positive Q fever (\%) & Positive chlamydiosis (\%) & Positive brucellosis (\%) \\
\hline Abortion & 70 & $30(42.85)$ & $14(20.00)$ & $6(8.57)$ \\
Abortion with arthritis & 1 & $1(100.00)$ & $0(0)$ & $0(0)$ \\
Orchitis & 3 & $2(66.67)$ & $0(0)$ & $0(0)$ \\
Repeat breeder & 6 & $4(66.67)$ & $0(0)$ & $1(16.67)$ \\
Sterile & 1 & $1(100)$ & $0(0)$ & $0(0)$ \\
Weak kids & 4 & $0(0)$ & $1(25.00)$ & $0(0)$ \\
Buck circulation & 33 & $17(51.51)$ & $3(9.09)$ & $1(3.03)$ \\
Total & 118 & $55(46.61)$ & $18(15.25)$ & $8(6.78)$ \\
\hline
\end{tabular}

TABLE 2: Univariate analysis of risk factors for bacterial infection-caused reproductive failures in smallholder goats herds in northeastern Thailand.

\begin{tabular}{|c|c|c|c|c|c|}
\hline Factor & Infected herd $(n=47)$ & Noninfected herd $(n=142)$ & OR & $95 \% \mathrm{CI}$ & $p$ value \\
\hline Group population (together) & 47 & 130 & 9.1 & $0.53-156.71$ & 0.1376 \\
\hline Buck circulation (use) & 47 & 66 & 109.29 & $6.61-1,807.38$ & 0.0010 \\
\hline Birth place (have) & 20 & 74 & 0.68 & $0.35-1.32$ & 0.2572 \\
\hline Brucellosis testing before movement (have) & 10 & 43 & 0.62 & $0.28-1.36$ & 0.2362 \\
\hline Movement (3 months) (have) & 42 & 116 & 1.88 & $0.68-5.22$ & 0.2241 \\
\hline Quarantine $30 \mathrm{~d}$ (have) & 6 & 22 & 0.8 & $0.3-2.11$ & 0.6488 \\
\hline Brucellosis testing annual (have) & 17 & 79 & 0.45 & $0.23-0.89$ & 0.0222 \\
\hline Personnel hygiene: mask (have) & 5 & 18 & 0.82 & $0.29-2.35$ & 0.7114 \\
\hline Personnel hygiene: glove birth (have) & 30 & 95 & 0.87 & $0.44-1.74$ & 0.6998 \\
\hline Personnel hygiene: handwashing (have) & 5 & 31 & 0.43 & $0.16-1.17$ & 0.0977 \\
\hline
\end{tabular}

Bold shows that significant association was classified as $p<0.05$. OR : odds ratio; CI : confidence interval.

show signs of reproductive failure, including abortion, miscarriage, stillbirths, and weak kids. In addition, orchitis, epididymis, and seminal vesiculitis can occur in bucks. Caprine brucellosis is caused by Gram-negative coccobacilli bacteria named Brucella melitensis. Regarding clinical signs, pregnant goats show placentitis and abortion in the second to third semesters or premature births, weak kids, a retained placenta, mastitis, metritis, repeat breeding, or infertility. Infected bucks may show signs of orchitis or epididymitis $[1,3]$. Other clinical signs may be sterility and arthritis [3].

Infected herds face economic losses due to the clinical signs of infection, such as abortion, infertility, still births, miscarriage in does, and sterile orchitis in bucks, which can reduce productivity. Moreover, zoonotic diseases can be transmitted to humans.

Information on bacterial infection causing reproductive failure in caprine was limited in northeast Thailand. The aim of this study is to determine the presence of antibodies against Q fever, chlamydiosis, and brucellosis, which can cause reproductive disorders in goats. Additionally, the risk factors associated with seropositivity in these infectious diseases are necessary for prevention and control measures due to their being zoonotic diseases.

\section{Materials and Methods}

2.1. Study Area. A retrospective study was conducted on the Caprine Brucellosis Surveillance Program being conducted by the Academic Service of the Faculty of Veterinary Medicine, Khon Kaen University, from March 2017 to September 2020. In total, 220 smallholder goat herds from 11 provinces including Buriram (4), Chaiyaphum (97), Khon
Kaen (59), Kalasin (2), Mahasarakham (13), Nongbualamphu (5), Nakhon Ratchasima (5), Roi Et (7), Sakon Nakhon (21), Srisakate (3), and Udon Thani (4) were included in services.

2.2. Sampling. Serum samples were collected from every goat aged over 4 months based on the criteria of the Brucellosis Surveillance Program [4, 5]. In total, 4,810 goats in 220 herds were tested for brucellosis by serological testing conducted by the Academic Service of the Faculty of Veterinary Medicine, Khon Kaen University.

Twenty-two percent (49/220) of smallholder goat herds were found to have clinical reproductive disorders in at least one case on farms in 8 provinces. A total of 118 were found to have reproductive disorders, such as abortion $(n=70)$, abortion with arthritis $(n=1)$, orchitis $(n=3)$, repeat breeding $(n=6)$, sterility $(n=1)$, and weak kids $(n=4)$, and bucks' circulation between herds $(n=33)$ was included in serological testing for $\mathrm{Q}$ fever and chlamydiosis.

2.3. Serological Tests. Blood samples were collected from the jugular vein into $5 \mathrm{ml}$ Vacutainer ${ }^{\circledR}$ red tubes. All samples were transported on ice to the laboratory at the Faculty of Veterinary Medicine, Khon Kaen University, within 6 h. Afterwards, all samples were centrifuged at $2,500 \mathrm{rpm}$ for $10 \mathrm{~min}$ for serum collection and then stored at $-20^{\circ} \mathrm{C}$ until analysis.

Screening for brucellosis was performed following the OIE standard procedure of the Rose Bengal technique, and the positive samples were confirmed by the complement fixation test [5].

IgG antibodies against C. burnetii and C. abortus infection were detected by the indirect ELISA IDEXX Q Fever 


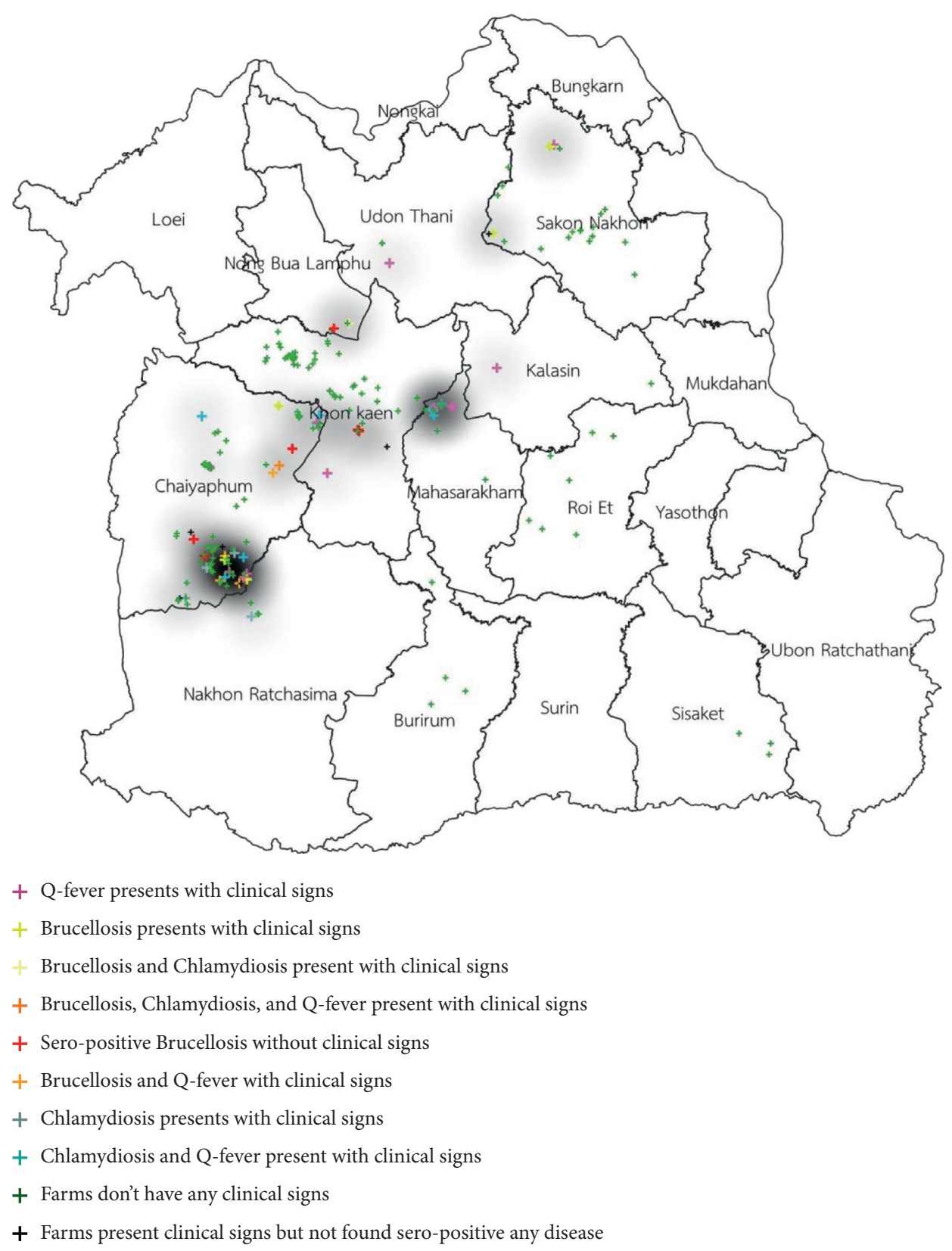

FIgURE 1: Distribution of clinical cases of brucellosis, chlamydiosis, and Q fever infection in smallholder meat goat herds in northeastern Thailand.

$\mathrm{Ab}$ test and IDEXX chlamydiosis Total Ab test (IDEXX Switzerland AG, Stationsstrasse 12, CH-3097 LiebefeldBern, Switzerland). The procedures were carried out according to the instructions of the manufacturer. The optical densities (OD) of the samples and controls were measured at $450 \mathrm{~nm}$ using a spectrophotometer. The S/P\% was determined based on the OD following the equation:

$$
\frac{S}{P \%}=100 \times \frac{\text { Sample } A(450)-\mathrm{NC} \bar{x} A(450)}{\mathrm{PC} \bar{x}-\mathrm{NC} \bar{x}} .
$$

The results were classified according to the manufacturer's instructions as follows.

IDEXX ELISA; S/P\% $>40,30<\mathrm{S} / \mathrm{P} \%<40$, and $\mathrm{S} / \mathrm{P} \%<30$ were positive, suspect, and negative samples, respectively.
The sensitivities of the test were $100 \%$ and $89-95 \%$ for the Q fever and chlamydiosis tests, respectively. The specificity of the test was $100 \%$ for both tests [6].

2.4. Questionnaires. One hundred eighty-nine owners were interviewed for their management of risk factors associated with the presence of disease. Factors included herd structure and herd and health management especially, history of buck in herd, movement and quarantine, personnel hygiene for feeding animals, barn cleaning, and giving birth to animals during parturition. Additionally, the herd's annual brucellosis testing was included in the questionnaires. 


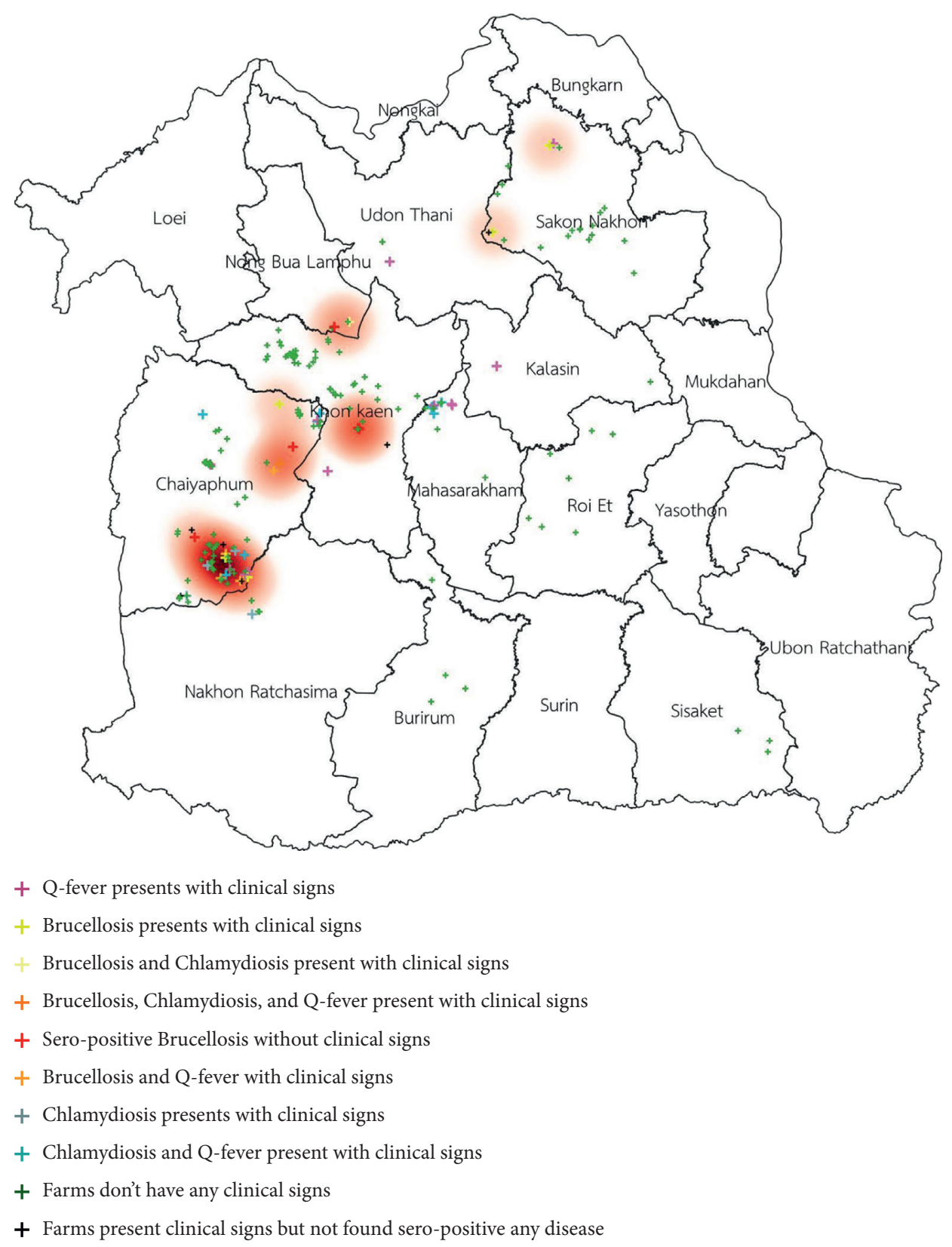

Figure 2: Distribution of clinical cases of brucellosis infection in smallholder meat goat herds in northeastern Thailand.

\subsection{Data Analysis}

2.5.1. Statistical Analysis. Data were analysed using Microsoft Office Excel 2016 and MedCalc ${ }^{\circledR}$ version 19.8 (MedCalc Software Ltd., 2021). The univariate analysis of risk factors was performed to identify variables associated with the positive herds at a significance level of 0.05 as the random effect in the model.

2.5.2. Epidemiological Analysis. The spatial distribution was analysed using Quantum GIS. The geographic coordinate system was used to produce the map for epidemiologic analysis.

\section{Results and Discussions}

3.1. Clinical Evidence Associated with Seropositivity and Spatial Distribution of Q Fever, Chlamydiosis, and Brucellosis in Meat Goat Herds. Based on clinical evidence of reproductive disorders in this area, 81.63\% (40/49) of the investigated herds already had bacterial infection due to seropositivity against $\mathrm{Q}$ fever, chlamydiosis, or brucellosis infection. Only 9 herds (9/49; $18.36 \%$ ) did not show antibodies against these bacterial infections by serological testing. Seropositivity to Q fever, chlamydiosis, and brucellosis testing in the herd was $45.0 \%(n=18 /$ $40), 25.0 \%(n=10 / 40)$, and $30.0 \%(n=12 / 40)$, respectively. In addition, 16 herds $(40.00 \%)$ had coinfection for at least two of three diseases. 


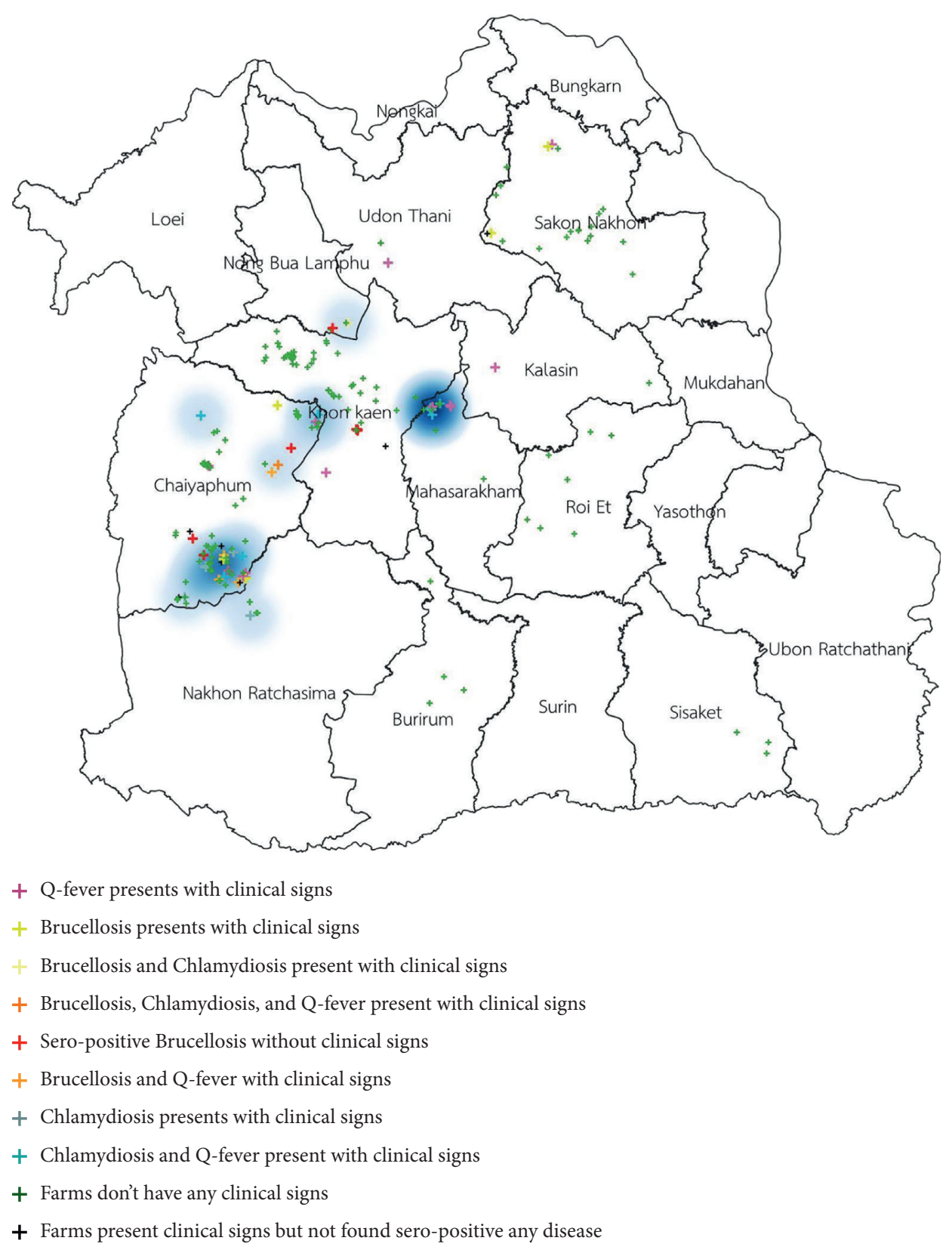

FIGURE 3: Distribution of clinical cases of chlamydiosis infection in smallholder meat goat herds in northeastern Thailand.

At the individual case level, seropositivity to $\mathrm{Q}$ fever, chlamydiosis, and brucellosis infection were $46.61 \%(55 / 118)$, $15.25 \%(18 / 118)$, and $6.78 \%(8 / 118)$, respectively. Abortion (71/ 118 ; 60.16\%) was the most common reproductive disorder in this area. Other clinical signs, such as orchitis, repeat breeding, sterility, and weak children were found in less than $15 \%$. Interestingly, 41 herds shared 33 circulations of bucks with other herds, and $60.6 \%$ of bucks (20/33) were seropositive with at least one of these infections. Eighty-five percent of infected bucks were seropositive for Q fever (17/20), followed by chlamydiosis in approximately $15 \%(3 / 20)$ and brucellosis at $5 \%(1 / 20)$. Seropositivity for brucellosis was the lowest in proportion in this area in comparison to $\mathrm{Q}$ fever and chlamydiosis. This might have been due to the active surveillance program for brucellosis, which screens for brucellosis annually in registered goat herds [7]. However, a few cases of reproductive failure caused by Brucella spp. still occurred in northeastern Thailand.

Clinical reproductive disorders and bucks' circulation related to serological testing are shown in Table 1.

3.2. Risk Factors. Herd and health management factors related to Q fever, chlamydiosis, and brucellosis infection were determined based on interviews with 189 owners of the 47 infected herds and 142 noninfected herds. All herds 


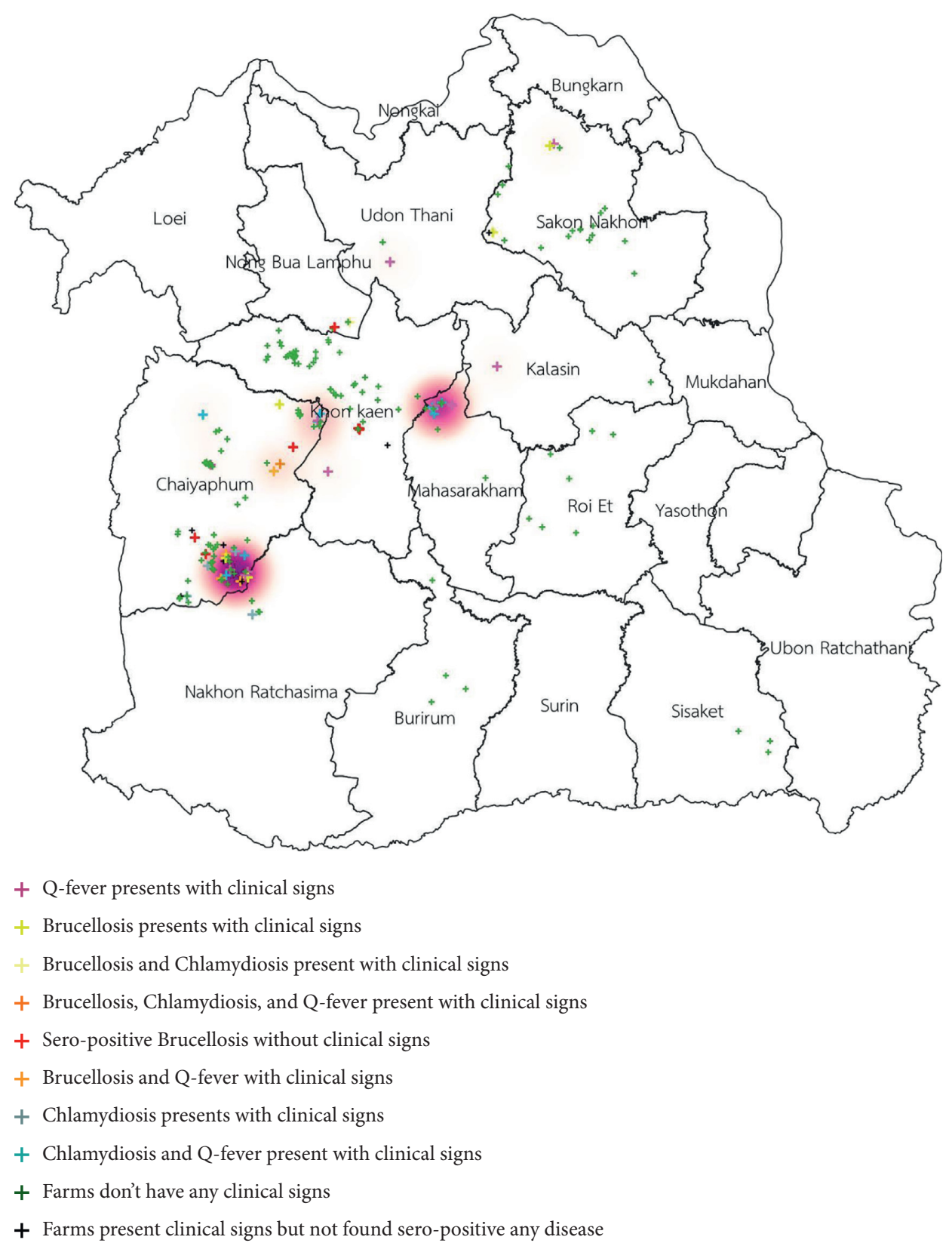

FIgURE 4: Distribution of clinical cases of Q fever in smallholder meat goat herds in northeastern Thailand.

used natural mating for breeding. Buck circulation between herds was a highly significant risk factor for seropositivity to at least one of those diseases in herds $(p=0.001$; odds ratio $(\mathrm{OR})=109.29 ; 95 \%$ confidence interval $(\mathrm{CI})=6.61-1,807.38)$. In addition, brucellosis testing annually was a highly significant protective factor for reproductive failure cases on farms $(p=0.022$; $\mathrm{OR}=0.45$; $95 \% \mathrm{CI}=0.23-0.89$; Table 2). Although other factors were not significantly associated with clinical reproductive disorders in this study, brucellosis testing before movement and quarantine at least 30 days before introduction to herds could reduce the opportunity for the introduction of pathogens from outside to the herds. Additionally, separating birth places from other areas and personnel hygiene during care of pregnant herds could protect farmers from pathogenic transmission while working on the farm.

3.3. Spatial Distribution of Q Fever, Chlamydiosis, and Brucellosis in Smallholder Goat Herds. The presence of reproductive disorder on farms related to bucks' circulation between herds was as follows: geographic information system data (GIS) showed that $93.93 \%$ of bucks (31/33) were seropositive to at least one Q fever, chlamydiosis, or brucellosis infection among the 41 herds.

The spatial distribution of three of those diseases is shown in Figure 1. The distribution of clinical cases of brucellosis, chlamydiosis, and Q fever is shown in Figures 2-4. The interviews showed that farmers preferred to use natural mating to 


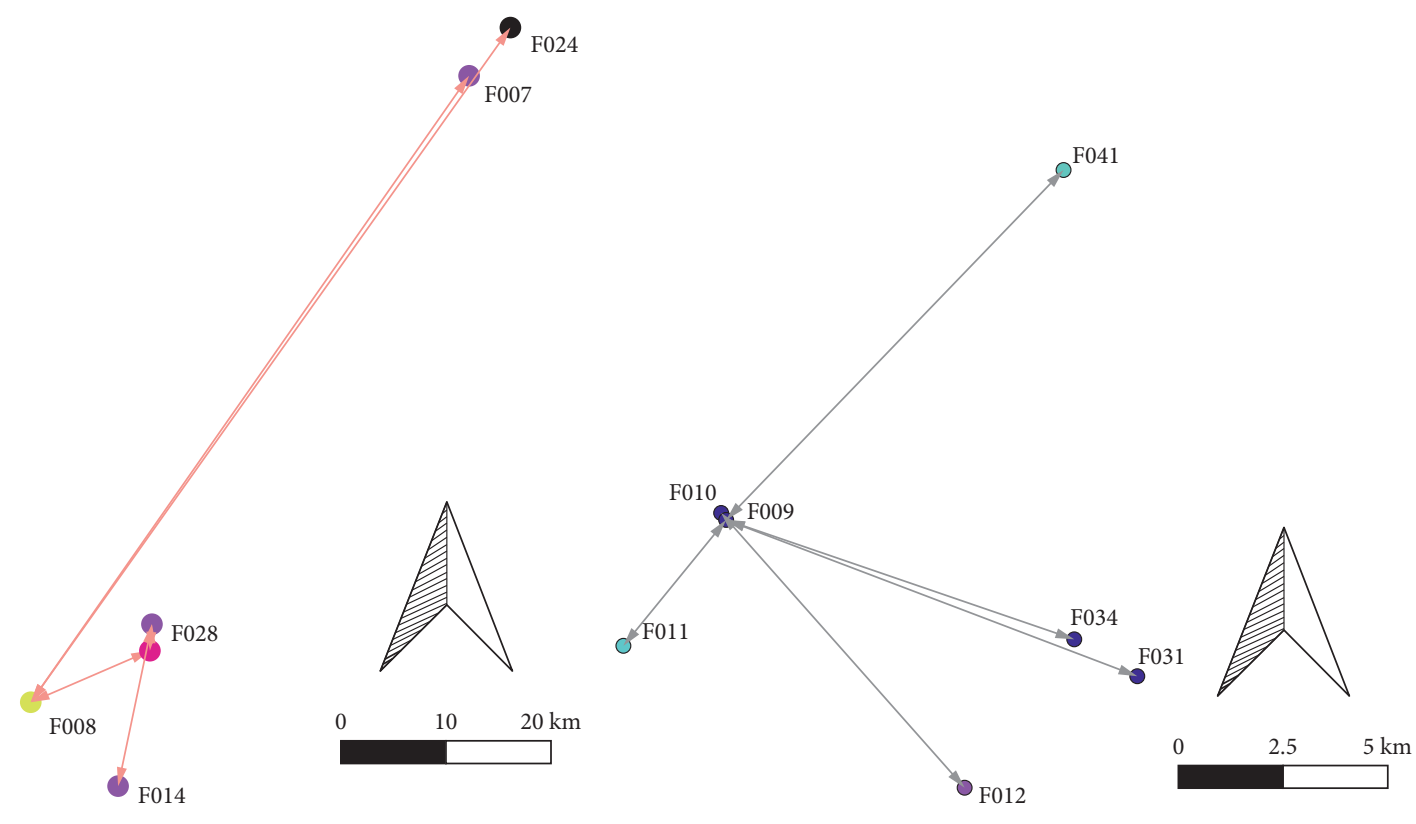

Cluster Brucellosis

Cluster Brucellosis +

Cluster Q-fever

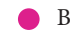
$\mathrm{BC}$
B
BQ
$\mathrm{NF}$
BQ
CQ
Q

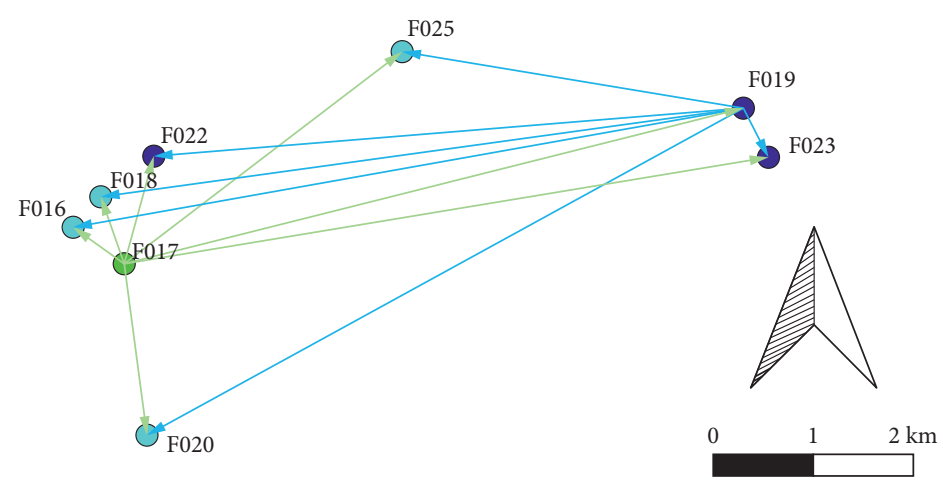

Cluster Q-fever

$\mathrm{C}$

$\mathrm{O}$ CQ

- Q

FIGURE 5: Cluster surge of brucellosis, Q fever, and chlamydiosis across herds within three communities due to shared buck circulation.

breed animals in their herds, and bucks' circulation between herds was commonly found in their communities. The GIS showed the cluster of Q fever-, chlamydiosis-, and brucellosisinfected herds that circulated the bucks in their community from one main herd to another (Figure 5). Frequently, bucks' circulation between herds not only spreads pathogens via natural mating with does, but pathogens can also spread to other healthy animals by inhalation, ingestion, and direct contact with contaminated material, such as faeces and urine of infected bucks. Most infected herds were coinfected with those diseases, and these might cause severe cases of reproductive disorders in goats.
Based on this investigation, the significant factor for controlling and preventing those diseases is avoiding bucks' circulation between herds. Although the serological screening of brucellosis is the only test in the Thai national brucellosis surveillance system, annual serological brucellosis testing was found to be a significant protective factor against bacterial reproductive disorders infection in herds. Additional advice for introducing animals, especially bucks, should be screening not only for brucellosis but also for $\mathrm{Q}$ fever and chlamydiosis.

Previous studies showed clinical cases of brucellosis and Q fever in humans related to livestock animals in Thailand 
[8-11]. Q fever, chlamydiosis, and brucellosis from small ruminants have been linked to zoonotic diseases between animals and humans and have caused serious human health problems worldwide [12-14]. In addition, these infections reduce the efficacy of animal health and production, decrease socioeconomic benefits, and increase the costs of human health care and communities resulting from the need for laboratory diagnosis, treatment, and control [15-20].

In northeastern Thailand, reproductive disorders have been reported to be caused by other diseases, such as epididymo-orchitis caused by Burkholderia pseudomallei [21], orchitis and mastitis caused by Corynebacterium pseudotuberculosis [22], abortion, still birth, and infertility with a low conception rate caused by Leptospira noguchii [23]. However, nine herds had reproductive failure and did not show antibodies against Q fever, chlamydiosis, brucellosis, melioidosis, caseous lymphadenitis, and leptospirosis. This might require further investigation for other bacterial infections, such as Campylobacter infection, listeriosis, and salmonellosis; other protozoa infections, such as neosporosis and toxoplasmosis; and virus infections such as Pestivirus infection.

\section{Data Availability}

Anyone can access the data by clicking on this link below https://docs.google.com/spreadsheets/d/1JE4OKLG7RAz RSm1BS8513nUri2rGRSnw/edit? usp=sharing

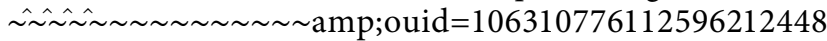

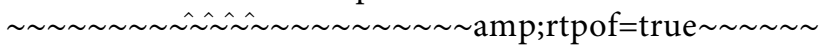

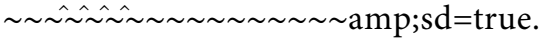

\section{Ethical Approval}

The study procedures were reviewed and approved by the Institutional Animal Care and Use Committee (record no. IACUC-KKU-83/60, date of approval 19 October 2017 and record no. IACUC-KKU-46/61, date of approval 16 August 2018). All cases were anonymised and aggregated to the village level. No personal identifiers were presented, and maps presented in this paper did not identify respondents' precise addresses.

\section{Conflicts of Interest}

The authors declare that they have no conflicts of interest.

\section{Authors' Contributions}

SR conceptualized the study, was involved in data collection, laboratory analysis, and statistical analysis, administered the project, and wrote the draft. SL and AS were involved in the epidemiological analysis. All authors commented and discussed the manuscript.

\section{Acknowledgments}

The authors wish to thank Monya Ekatat, DVM, from the National Institute of Animal Health for their extensive knowledge and suggestions about the update regarding brucellosis in small ruminants in Thailand. Special thanks are due to Pichet Thongpun and Udom Chuachan, DVM, from the Veterinary Research and Development Center Upper and Lower Northeastern Region for screening and confirming the brucellosis. The authors would also like to thank the farmers involved in the study for their cooperation. This work was financially supported by the Bureau of Academic Services, Khon Kaen University from 2017 to 2020, and the group of Animal Technology, Faculty of Veterinary Medicine, Khon Kaen University.

\section{References}

[1] M. C. Smith and D. M. Sherman, Goat Medicine, Wiley-Blackwell, Hoboken, NY, USA, 2nd edition, 2009.

[2] S. R. Porter, G. Czaplicki, J. Mainil, R. Guattéo, and C. Saegerman, "Q fever: current state of knowledge and perspectives of research of a neglected zoonosis," International Journal of Microbiology, vol. 2011, Article ID 248418, 22 pages, 2011.

[3] World Health Organization (WHO), "Brucellosis in humans and animals," 2006, https://www.who.int/csr/resources/ publications/Brucellosis.pdf?ua $=1$.

[4] Royal Thai Government Gazette, "Notification of the department of livestock development. National animal disease surveillance system. [online].Retrieved from,” 2019, http:// www.ratchakitcha.soc.go.th/DATA/PDF/2562/E/047/T_0041. PDF.

[5] OIE Terrestrial Manual, "Brucellosis (Brucella abortus, B.melitensis and B.suis)," 2018, https://www.oie.int/fileadmin/Home/ eng/Health_standards/tahm/3.01.04_BRUCELLOSIS.pdf.

[6] IDEXX Validation report, IDEXX Q Fever Ab Test (IDEXX Switzerland AC, Stationsstrasse 12, CH-3097 Liebefeld-Bern, Switzerland) and IDEXX Chlamydiosis Total Ab Test (IDEXX Switzerland AG, Stationsstrasse 12, CH-3097 Liebefeld-Bern, Switzerland), 2011.

[7] Department of Livestock Development (DLD) National Animal disease surveillance System, "Brucellosis surveillance in small ruminants," 2020, https://drive.google.com/file/d/ 1gOl5PAgQCfmzgUDe132UgVGSRhXA0hS7/view.

[8] C. Ekpanyaskul, S. Santiwattanakul, W. Tantisiriwat, and W. Buppanharun, "Factors associated with seropositive antibodies to Brucella melitensis in the Nakhon Nayok, Thailand," Journal of the Medical Association of Thailand, vol. 95, no. Suppl 12, pp. S40-S46, 2012.

[9] O. Pachirat, P.-E. Fournier, B. Pussadhamma et al., "The first reported cases of Q fever endocarditis in Thailand," Infectious Disease Reports, vol. 4, no. 1, pp. 17-18, 2012.

[10] K. Lapphral, A. Leelaporn, N. Vanprapar et al., "Case report: first case report of Brucellosis in a child in Thailand," The Southeast Asian Journal of Tropical Medicine and Public Health, vol. 45, no. 4, pp. 890-896, 2014.

[11] J. M. Lewis, J. Folb, S. B. Kalra, M. Taegtmeyer, and N. J. Beechinga, "Brucella melitensis prosthetic joint infection in a traveler returning to the UK from Thailand: case report and review of the literature," Travel Medicine and Infectious, vol. 14, 2016.

[12] J. H. McQuiston and J. E. Childs, "Q fever in humans and animals in the United States," Vector Borne and Zoonotic Diseases, vol. 2, no. 3, pp. 179-191, 2002.

[13] A. Tesfaye, M. Sahele, T. Sori, C. Guyassa, and A. Garoma, "Seroprevalence and associated risk factors for chlamydiosis, coxiellosis and brucellosis in sheep and goats in Borana 
pastoral area, southern Ethiopia," BMC Veterinary Research, vol. 16, no. 1, pp. 1-8, 2020.

[14] “OIE Distribution maps," 2019, https://www.oie.int/wahis_2/ public/wahid.php/Diseaseinformation/Diseasedistributionmap.

[15] J. A. van Loenhout, W. J. Paget, J. H. Vercoulen, C. J. Wijkmans, J. L. Hautvast, and K. van der Velden, "Assessing the long-term health impact of Q-fever in The Netherlands: a prospective cohort study started in 2007 on the largest documented Q-fever outbreak to date," BMC Infectious Diseases, vol. 12, no. 1, p. 280, 2012.

[16] H. M. Oboge, "Socio-economics and perceptions of Q-fever infection in a pastoralist system of Kajiado County," MSc thesis, University of Nairobi, Nairobi, Kenya, 2016.

[17] A. Robertson, I. Handel, and N. D. Sargison, "General evaluation of the economic impact of introduction of Chlamydia abortus to a Scottish sheep flock," Veterinary Record Case Reports, vol. 6, no. 3, Article ID e000689, 2018.

[18] B. Megersa, D. Biffa, F. Abunna, A. Regassa, J. Godfroid, and E. Skjerve, "Seroprevalence of brucellosis and its contribution to abortion in cattle, camel and goat kept under pastoral management in Borana, Ethiopia," Tropical Animal Health and Production, vol. 43, no. 3, pp. 651-656, 2011.

[19] B. B. Singh, N. K. Dhand, and J. P. S. Gill, "Short communication: economic losses occurring due to brucellosis in Indian livestock populations," Preventive Veterinary Medicine, vol. 119, no. 3-4, pp. 211-215, 2015.

[20] K. A. Franc, R. C. Krecek, B. N. Häsler, and A. M. ArenasGamboa, "Brucellosis remains a neglected disease in the developing world: a call for interdisciplinary action," $B M C$ Public Health, vol. 18, no. 1, pp. 1-9, 2018.

[21] S. Rerkyusuke, C. Potisap, R. W. Sermswan, S. Wongratanacheewin, S. Tangkawattana, and P. Thongpun, "Case report: testicular abscess caused by Burkholderia pseudomallei in a buck goat," in Proceedings of the Chiang Mai University Veterinary Conference, Chiang Mai, Thailand, December 2020.

[22] S. Thongkwow, N. Poosiripinyo, N. Pongkornkumpon et al., "Distribution and risk factors of clinical caseous lymphadenitis in small-holder goat herds in Northeastern Thailand," Thai Journal Veterinary Medicine, vol. 49, no. 4, pp. 343-351, 2019.

[23] S. Rerkyusuke, S. Chaimongkol, N. Fungbun, P. Phuektes, and D. Suwancharoen, "Reproductive failures caused by Leptospira noguchii in meat goat," in Proceedings of the Chiang Mai University Veterinary Conference, Chiang Mai, Thailand, December 2020. 